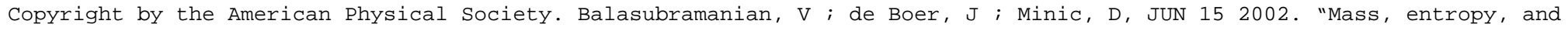

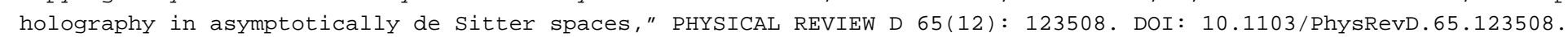

PHYSICAL REVIEW D, VOLUME 65, 123508

\title{
Mass, entropy, and holography in asymptotically de Sitter spaces
}

\author{
Vijay Balasubramanian* \\ David Rittenhouse Laboratories, University of Pennsylvania, Philadelphia, Pennsylvania 19104 \\ Jan de Boer $^{\dagger}$ \\ Instituut voor Theoretische Fysica, Valckenierstraat 65, 1018XE Amsterdam, The Netherlands \\ Djordje Minic ${ }^{\ddagger}$ \\ Institute for Particle Physics and Astrophysics, Department of Physics, Virginia Tech, Blacksburg, Virginia 24061
}

(Received 30 January 2002; published 3 June 2002)

\begin{abstract}
We propose a novel prescription for computing the boundary stress tensor and charges of asymptotically de Sitter (dS) spacetimes from data at early or late time infinity. If there is a holographic dual to dS spaces, defined analogously to the AdS/conformal field theory correspondence, our methods compute the (Euclidean) stress tensor of the dual. We compute the masses of Schwarzschild-de Sitter black holes in four and five dimensions, and the masses and angular momenta of Kerr-de Sitter spaces in three dimensions. All these spaces are less massive than de Sitter space, a fact which we use to qualitatively and quantitatively relate de Sitter entropy to the degeneracy of possible dual field theories. Our results in general dimensions lead to a conjecture: Any asymptotically de Sitter spacetime with mass greater than de Sitter space has a cosmological singularity. Finally, if a dual to de Sitter space exists, the trace of our stress tensor computes the renormalized group (RG) equation of the dual field theory. Cosmological time evolution corresponds to RG evolution in the dual. The $\mathrm{RG}$ evolution of the $c$ function is then related to changes in accessible degrees of freedom in an expanding universe.
\end{abstract}

DOI: 10.1103/PhysRevD.65.123508

PACS number(s): $98.80 . \mathrm{Hw}$

\section{INTRODUCTION}

There is no good local notion of energy in a gravitating spacetime. Nevertheless, there is a notion of mass, or total energy, which can be computed from the effects of matter on spacetime geometry [1]. Heuristically, the deviation of the metric and other fields near spatial infinity from their form in the vacuum provides a measure of mass, angular momentum and other conserved charges. Equivalently, these charges can be computed from the asymptotic symmetries of a spacetime; e.g., the eigenvalue of an asymptotic timelike Killing vector will give a measure of mass.

There are two basic obstacles to applying this wellunderstood philosophy to de Sitter space. First, there is no spatial infinity - the asymptotic regions of de Sitter space are Euclidean surfaces at early and late temporal infinity $\left(\mathcal{I}^{ \pm}\right)$ [2]. Second, there is no asymptotic Killing vector that is globally timelike.

In this article, we will evade these obstacles by computing the quasilocal stress tensor of Brown and York [3] on the Euclidean surfaces at $\mathcal{I}^{ \pm}$, and by using this quantity to define a novel notion of mass, and other charges appropriate to the asymptotic symmetries. ${ }^{1}$ Our methods are strongly remi-

\footnotetext{
*Electronic address: vijay@endive.hep.upenn.edu

${ }^{\dagger}$ Electronic address: jdeboer@wins.uva.nl

‡Electronic address: dminic@vt.edu

${ }^{1}$ Other interesting approaches to defining mass in de Sitter space include [4] and [5] which investigate possible positive mass theorems. The latter works define mass by using timelike conformal Killing vectors of de Sitter space.
}

niscent of the computation of conserved charges in a conformal field theory (CFT). If de Sitter space is dual to a Euclidean CFT [6-10] defined in a manner analogous to the AdS/ CFT correspondence $[11,12]$ we are computing the energy and charges of states of the dual.

We use our stress tensor to display the asymptotic conformal isometries of $\mathrm{dS}_{3}$ and compute the central charge $(c)$ of the symmetry algebra. In three dimensions, with a positive cosmological constant, there are no black holes, but there is a spectrum of spinning conical defects [13]. We derive these solutions as quotients of $\mathrm{dS}_{3}$ and then compute their masses $(M)$ and angular momenta $(J)$. The conical defects are less massive than de Sitter space. Remarkably, naively entering $c$, $M$, and $J$ into the Cardy formula of a hypothesized 2D CFT dual exactly reproduces the entropy of the cosmological horizon of these spaces. Related uses of the Cardy formula to study $\mathrm{dS}_{3}$ entropy appear in work by Park [13] and, more recently, [14]. Here we point out several subtleties that face this reasoning, and some potential resolutions. ${ }^{2}$

We proceed to compute the masses of the Schwarzschild-de Sitter black holes in 4 and 5 dimensions. (See [15] for related discussions.) We find that de Sitter space is more massive than the black hole spacetimes. In fact, this is a qualitative feature necessary for de Sitter entropy to have an interpretation in terms of the degeneracy of a dual field theory defined in the AdS/CFT mode. As Bousso has emphasized, the entropy of de Sitter space is an upper bound on the entropy of any asymptotically de Sitter space-

\footnotetext{
${ }^{2}$ Other approaches to de Sitter entropy have been suggested in [16-19].
} 
time [20]. Since field theories generically have entropies that increase with energy, a dual description of dS entropy should map larger Schwarzschild-dS black holes into states of lower energy, precisely as we find here. Indeed, the largest black hole in de Sitter space (the Nariai black hole [21]) has the least mass.

Following Bousso [20] we expect that asymptotically de Sitter spaces with entropy greater than de Sitter entropy do not exist. Therefore, since our mass formula increases monotonically with entropy, we arrive at a conjecture: ${ }^{3}$ Any asymptotically de Sitter space whose mass exceeds that of de Sitter space contains a cosmological singularity.

Given a holographic dual to asymptotically de Sitter space, the trace of our stress tensor computes the dual renormalization group (RG) equation. We therefore arrive at a remarkable picture: RG evolution of the dual is time evolution in an expanding universe. ${ }^{4}$ The evolution of the central charge in the dual is directly related to the changing number of accessible degrees of freedom in spacetime. The field theory $c$-theorem is related to properties of the Raychaudhuri equation in gravity, in analogy with the properties of holographic RG flows in the AdS/CFT correspondence [23-26].

We conclude the paper with a discussion of prospects for finding a holographic dual to de Sitter space. The material in this article has been presented at a variety of conferences and workshops (see the Acknowledgments). While the text was being prepared, we received a number of unpublished works addressing various related aspects of de Sitter physics [27].

\section{BOUNDARY STRESS TENSOR, MASS AND CONSERVED CHARGES}

To date there is no completely adequate proposal for measuring the mass of asymptotically de Sitter spaces. The classic work of Abbott and Deser remains the basic technique [28], but is a perturbative approach measuring the energy of fluctuations. In AdS space, the Brown-York boundary stress tensor [3], augmented by counterterms inspired by the duality with a CFT [29-31], led to a particularly convenient method of computing the mass of asymptotically AdS spacetime. Here we argue that a similar approach applies to de Sitter space.

In $d+1$ dimensions, spaces with a positive cosmological constant solve equations of motions derived from the action

$$
\begin{aligned}
I_{B}= & -\frac{1}{16 \pi G} \int_{\mathcal{M}} d^{d+1} x \sqrt{-g}(R+2 \Lambda) \\
& +\frac{1}{8 \pi G} \int_{\partial \mathcal{M}^{-}}^{\partial \mathcal{M}^{+}} d^{d} x \sqrt{h} K .
\end{aligned}
$$

\footnotetext{
${ }^{3}$ As we will see below, we could have chosen to define the classical stress tensor of de Sitter space with the opposite sign. With this definition, we would find that de Sitter space has a lower mass than the black hole spacetimes and our conjecture becomes a positive mass conjecture for non-singular asympototically de Sitter spacetimes, i.e., that de Sitter space itself has the lowest mass among such spaces.

${ }^{4}$ As this paper was being typed, the same point was made by Strominger [22].
}

Here $\mathcal{M}$ is the bulk manifold, $\partial \mathcal{M}^{ \pm}$are spatial boundaries at early and late times, $g_{i j}$ is the metric in the bulk of spacetime, and $h_{\mu \nu}$ and $K$ are the induced metric and the trace of the extrinsic curvature of the boundaries. In de Sitter space the spacetime boundaries $\mathcal{I}^{ \pm}$are Euclidean surfaces at early and late time infinity. The notation $\int_{\partial \mathcal{M}}^{\partial \mathcal{M}^{+}} d^{2} x$ indicates an integral over the late time boundary minus an integral over the early time boundary which are both Euclidean surfaces. ${ }^{5}$ The extrinsic curvature boundary term is necessary to allow a well-defined Euler-Lagrange variation.

It will be convenient to define a length scale

$$
l=\sqrt{\frac{d(d-1)}{2 \Lambda}} .
$$

In terms of $l$, the vacuum de Sitter solution to equations of motion derived from Eq. (1) is

$$
d s^{2}=-d t^{2}+l^{2} \cosh ^{2}(t / l)\left(d \Omega_{d}^{2}\right)
$$

where equal time sections are $d$-spheres. The same space admits a coordinate system where equal time surfaces are flat:

$$
d s^{2}=-d \tau^{2}+e^{2 \tau / l} d \vec{x}^{2}=\frac{l^{2}}{\eta^{2}}\left[-d \eta^{2}+d \vec{x}^{2}\right],
$$

with $\tau \in[-\infty,+\infty]$ while $\eta \in(0, \infty]$. This patch only covers half of de Sitter space, extending from a "big bang" at a past horizon to the Euclidean surface at future infinity. By replacing $\tau$ by $-\tau$ a patch which covers the other half of de Sitter space (from past infinity to a future horizon) can be obtained. We will refer to these two patches as the "big bang" and the "big crunch" patches. Finally, an inertial observer in de Sitter space sees a static spacetime with a cosmological horizon:

$$
d s^{2}=-\left(1-\frac{r^{2}}{l^{2}}\right) d t^{2}+\left(1-\frac{r^{2}}{l^{2}}\right)^{-1} d r^{2}+r^{2} d \Omega_{d-1}^{2}
$$

The relations between these coordinate patches and Penrose diagrams are presented in Hawking and Ellis [2] and references therein.

We can formally "Wick rotate" from a positive to a negative cosmological constant by the analytic continuation $l$ $\rightarrow i l$. This formal transformation (sometimes accompanied by additional Wick rotations of some of the coordinates) takes patches of de Sitter into patches of anti-de Sitter space. For example, the static path (5) rotates to global AdS. Likewise, redefining $e^{\tau / l}=r / l$ and carrying out some formal analytic continuations gives the Poincare patch of AdS. Tracking these continuations through the classic computations of properties of AdS spaces gives a powerful method of inferring some aspects of de Sitter physics.

\footnotetext{
${ }^{5}$ We follow the conventions of Brown and York [3] for the boundary terms and those of [30] for the bulk term.
} 


\section{A. A finite action}

In general the action (1) is divergent when evaluated on a solution to the equations of motion, because of the large volume at early and late times. For example, the late time region of the inflationary big bang patch makes a divergent contribution to the action. Specifically, if we cut time off at a large time $t$, the leading term in the 3 dimensional action $(d+1=3)$ is

$$
I=\frac{1}{8 \pi G} \int d^{2} x e^{2 t / l}\left(\frac{-1}{l}\right)+\text { finite }
$$

which diverges as $t \rightarrow \infty$. (The same applies to the early time region of the inflationary big crunch patch.) Mottola and Mazur [33] have shown that the results of Fefferman and Graham [34] and Henningson and Skenderis [29] on the asymptotics of spaces with a negative cosmological constant can be extended to spaces with a positive cosmological constant. Combining their results with the reasoning in $[29,30]$ shows that the divergences of the action (1) can be canceled by adding local boundary counterterms that do not affect the equations of motion.

For example, in three dimensions the improved action

$$
\begin{aligned}
I= & I_{B}+\frac{1}{8 \pi G} \int_{\partial \mathcal{M}^{+}} d^{2} x \sqrt{h} \frac{1}{l} \\
& +\frac{1}{8 \pi G} \int_{\partial \mathcal{M}^{-}} d^{2} x \sqrt{h} \frac{1}{l}
\end{aligned}
$$

has the same solutions as Eq. (1) but is finite for asymptotically de Sitter spaces. Indeed, the counterterms in Eq. (7) clearly cancel the divergent terms of the bare action in inflationary coordinates. In analogy with $\mathrm{AdS}$, if we place boundary conditions requiring asymptotically $\mathrm{dS}$ spaces to approach the de Sitter background sufficiently quickly at early and late times, the divergences of the classical action will cancel.

In all dimensions the action has a class of divergences that are powers of the conformal time coordinate $\eta$ appearing in Eq. (4). In 3, 4 and 5 dimensions these are canceled by the counterterms

$$
\begin{gathered}
I_{\mathrm{ct}}=\frac{1}{8 \pi G} \int_{\partial \mathcal{M}^{+}} d^{2} x \sqrt{h} L_{\mathrm{ct}}+\frac{1}{8 \pi G} \int_{\partial \mathcal{M}^{-}} d^{2} x \sqrt{h} L_{\mathrm{ct}}, \\
L_{\mathrm{ct}}=\frac{(d-1)}{l}-\frac{l^{2}}{2(d-2)} R .
\end{gathered}
$$

The second counterterm only applies when $d+1>3$. Here $R$ is the intrinsic curvature of the boundary surface, and calculations are performed by cutting off de Sitter space at a finite time, and then letting the surface approach infinity. In odd dimensions there is one additional divergence that is logarithmic in the conformal time $\eta$. This divergence cannot be canceled without including an explicit cutoff dependence in the counterterm action, thereby leading to a conformal anomaly. In the situations we study the anomaly and the associated logarithmic divergence vanish and so we will ne- glect it here. See $[29,32]$ for a detailed discussion of this issue in the framework of the AdS/CFT correspondence. It would be interesting to repeat the analysis of [35] to derive the counterterms (9) for general dimensions from the GaussCodazzi equations for spaces with a positive cosmological constant. Likewise, the analysis of logarithmic divergences in [32] should be extended to de Sitter space.

\section{B. Boundary stress tensor}

In AdS space, the Brown-York prescription [3,30] was used to compute a quasilocal boundary stress tensor that measures the response of the spacetime to changes of the boundary metric. We can carry out an analogous procedure in de Sitter space to compute a Euclidean boundary stress tensor on the spacetime boundary. First, write the spacetime metric in Arnowitt-Deser-Misner (ADM) form as

$$
\begin{aligned}
d s^{2} & =g_{i j} d x^{i} d x^{j} \\
& =-N_{t}^{2} d t^{2}+h_{\mu \nu}\left(d x^{\mu}+V^{\mu} d t\right)\left(d x^{\nu}+V^{\nu} d t\right) .
\end{aligned}
$$

Then $h_{\mu \nu}$ is the metric induced on surfaces of fixed time, and choosing $u^{\mu}$ to be the future pointing unit normal to these surfaces, we can compute the extrinsic curvature

$$
K_{\mu \nu}=-h_{\mu}^{i} \nabla_{i} u_{\nu}
$$

and its trace $K$. (Here the index on $h_{\mu \nu}$ is raised by the full metric $g_{i j}$.) The Euclidean quasilocal stress tensor of de Sitter space is given by the response of the action, evaluated on the space of classical solutions, to variations of the boundary metric. We can evaluate these variations either on an early or late time boundary, getting the stress tensors

$$
\begin{aligned}
T^{+\mu \nu}= & \frac{2}{\sqrt{h}} \frac{\delta I}{\delta h_{\mu \nu}} \\
= & -\frac{1}{8 \pi G}\left[K^{\mu \nu}-K h^{\mu \nu}-\frac{(d-1)}{l} h^{\mu \nu}\right. \\
& \left.-\frac{l}{(d-2)} G^{\mu \nu}\right], \\
T^{-\mu \nu}= & \frac{2}{\sqrt{h}} \frac{\delta I}{\delta h_{\mu \nu}} \\
= & -\frac{1}{8 \pi G}\left[-K^{\mu \nu}+K h^{\mu \nu}-\frac{(d-1)}{l} h^{\mu \nu}\right. \\
& \left.-\frac{l}{(d-2)} G^{\mu \nu}\right],
\end{aligned}
$$

where the term proportional to $G^{\mu \nu}$, the Einstein tensor of the boundary geometry, only appears when $d+1>3$. The last two terms in Eqs. (12) and (13) come from variation of the counterterms in Eq. (8). To obtain the boundary stress tensor we evaluate Eq. (12) at fixed time and send the time to infinity so that the surface approaches the spacetime boundary. The two Eqs. (12) and (13) appear to give different stress 
tensors because of the relative signs of terms. In fact, in empty de Sitter space they are evaluating identical quantities - the difference in signs occurs because the extrinsic curvature $K$ is evaluated with respect to a future pointing timelike normal leading to some sign changes between early and late time surfaces. For this reason, we will simply drop the \pm and use $T_{\mu \nu} \equiv T^{+\mu \nu}$ in the examples we study. Note also that there are some sign differences between Eq. (12) and the quasilocal stress tensor in AdS space [30]. These arise, following Brown and York [3], from some differences between the treatment of timelike and spacelike boundaries.

Since we are working on the Euclidean surface at $\mathcal{I}^{ \pm}$, we could equally well have chosen to define the stress tensors in Eqs. (12) and (13) with the opposite sign as $(-2 / \sqrt{h}) \delta I / \delta h_{\mu \nu}$. This alternative sign does not change any essential physics—we will point out the slightly different interpretations that follow in various places.

It is worth working out some examples of the stress tensor which we will have use of later. An equal time surface of the inflating patch (4) in 3 dimensions is the infinite Euclidean plane. Evaluating the stress tensor (12) on this surface gives

$$
T^{\mu \nu}=-\frac{1}{8 \pi G l} e^{-2 t / l}+\frac{1}{8 \pi G l} e^{-2 t / l}=0 .
$$

Here the bare stress tensor canceled exactly against the counterterm. By contrast, in global coordinates, the boundary stress tensor of $\mathrm{dS}_{3}$ is

$$
\begin{gathered}
T^{\mu \nu}=\frac{e^{-t / l}}{8 \pi G l^{3} \cosh ^{3}(t / l)}\left(\begin{array}{cc}
1 & 0 \\
0 & 1 / \sin ^{2} \theta
\end{array}\right), \\
T=\frac{1}{4 \pi G l} \frac{e^{-t / l}}{\cosh (t / l)}
\end{gathered}
$$

where $T$ is the trace of the stress tensor. Notice that the stress tensor vanishes exponentially for $t \rightarrow \infty$.

\section{Mass and other conserved charges}

In a theory of gravity, mass is a measure of how much a metric deviates near infinity from its natural vacuum behavior; i.e., mass measures the warping of space. The boundary stress tensor (12) computes the response of the spacetime action to such a warping and thereby encodes a notion of mass. Inspired by the analogous reasoning in AdS space $[3,30]$, we propose a notion of mass for an asymptotically de Sitter space. We can always write the metric $h_{\mu \nu}$ on equal time surfaces in the form

$$
\begin{aligned}
h_{\mu \nu} d x^{\mu} d x^{\nu}= & N_{\rho}^{2} d \rho^{2}+\sigma_{a b}\left(d \phi^{a}+N_{\Sigma}^{a} d \rho\right) \\
& \times\left(d \phi^{b}+N_{\Sigma}^{b} d \rho\right)
\end{aligned}
$$

where the $\phi^{a}$ are angular variables parametrizing closed surfaces around an origin. Let $\xi^{\mu}$ be a Killing vector generating an isometry of the boundary geometry. Following $[3,30]$ we can define the conserved charge associated to $\xi^{\mu}$ as follows. Let $n^{\mu}$ be the unit normal on a surface of fixed $\rho$ and define the charge

$$
Q=\oint_{\Sigma} d^{d-1} \phi \sqrt{\sigma} n^{\mu} \xi^{\mu} T_{\mu \nu}
$$

In our computation $\rho$ will always be the coordinate associated with the asymptotic Killing vector that is timelike inside the static patch, but spacelike at $\mathcal{I}^{ \pm}$. It would be interesting to compare this notion of a conserved charge in de Sitter space to that defined by Abbott and Deser [28].

An important difficulty facing the definition of mass in de Sitter space is the absence of a globally timelike Killing vector. However, as is evident from Eq. (5), there is a Killing vector that is timelike inside the static patch, while it is spacelike outside the cosmological horizon and therefore on $\mathcal{I}^{ \pm}$. Any space that is asymptotically de Sitter will have such an asymptotic symmetry generator. We can adapt the coordinates (16) so that "radial" normal $n^{\mu}$ is proportional to the relevant (spacelike) boundary Killing vector $\xi^{\mu}$. Then, we propose that an interesting and useful notion of the mass $M$ of an asymptotically de Sitter space is:

$$
M=\oint_{\Sigma} d^{d-1} \phi \sqrt{\sigma} N_{\rho} \epsilon, \quad \epsilon \equiv n^{\mu} n^{\nu} T_{\mu \nu},
$$

where we normalized the Killing vector in Eq. (17) as $\xi^{\mu}$ $=N_{\rho} n^{\mu}$. Likewise, we can define momenta

$$
P_{a}=\oint_{\Sigma} d^{d-1} x \sqrt{\sigma} j_{a}, \quad j_{a}=\sigma_{a b} n_{\mu} T^{a \mu}
$$

We compute this formula on a surface of fixed time and then send time to infinity so that it approaches the spacetime boundaries at $\mathcal{I}^{ \pm}$.

Below we will investigate the meaning of the de Sitter stress tensor and conserved charges in various dimensions.

\section{THREE DIMENSIONAL COSMOLOGICAL SPACETIMES}

\section{A. More on classical solutions}

We seek an interesting class of solutions to $2+1$ dimensional gravity with a positive cosmological constant on which to test our stress tensor and definition of mass. In 3 dimensions black holes only exist when there is a negative cosmological constant, but when $\Lambda>0$ we can find a class of spinning conical defects which we will refer to as the Kerr-de Sitter spaces following Park [13]. These spaces have been discussed before (see, e.g., $[36,13,17]$ ), but we will derive them below as quotients of $\mathrm{dS}_{3}$. For convenience we will set the de Sitter scale to $1(l=1)$ and will restore it later by dimensional analysis.

Three dimensional de Sitter space is the quotient $S L(2, C) / S L(2, R)$, and any solution of the field equations looks locally the same. Thus the general solution will locally look like $S L(2, C) / S L(2, R)$, but can be subject to various global identifications. In particular, we can consider solutions of the form $\Gamma \backslash S L(2, C) / S L(2, R)$ for some discrete subgroup $\Gamma$ of $S L(2, C)$. If the discrete subgroup is generated by a single element of $S L(2, C)$, there are two possibilities. Up to 
conjugation, the generator can be of the form

$$
\left(\begin{array}{cc}
q & 0 \\
0 & q^{-1}
\end{array}\right) \quad \text { or } \quad\left(\begin{array}{ll}
1 & a \\
0 & 1
\end{array}\right)
$$

The second case appears to correspond to solutions that look like the inflationary patch (4), but with the complex plane replaced by a cylinder. We will restrict our attention to solutions that correspond to identifications of the first type.

An element of $\mathrm{dS}_{3}=S L(2, C) / S L(2, R)$ can be written as

$$
M=\left(\begin{array}{cc}
u & i \alpha \\
i \beta & \bar{u}
\end{array}\right), \quad u \bar{u}+\alpha \beta=1, \quad \alpha, \beta \in R,
$$

in terms of which the metric is simply

$$
d s^{2}=d u d \bar{u}+d \alpha d \beta .
$$

$S L(2, C)$ acts on $M$ as

$$
M \rightarrow\left(\begin{array}{ll}
a & b \\
c & d
\end{array}\right) M\left(\begin{array}{cc}
\bar{d} & -\bar{b} \\
-\bar{c} & \bar{a}
\end{array}\right)
$$

Setting $q=\exp \left(\pi\left(r_{-}+i r_{+}\right)\right)$, the identifications $\Gamma$ imposes on $\mathrm{dS}_{3}$ are, in terms of the two by two matrix parametrization, given by

$$
\left(\begin{array}{cc}
u & i \alpha \\
i \beta & \bar{u}
\end{array}\right) \sim\left(\begin{array}{cc}
e^{2 \pi i r_{+}} u & i e^{2 \pi r_{-}} \alpha \\
i \beta e^{-2 \pi r_{-}} & \bar{u} e^{-2 \pi i r_{+}}
\end{array}\right) .
$$

To achieve such an identification, we parametrize the two by two matrix as

$$
\left(\begin{array}{cc}
u & i \alpha \\
i \beta & \bar{u}
\end{array}\right)=\left(\begin{array}{cc}
e^{i r_{+} \phi} \sqrt{1-\alpha \beta} & i e^{r_{-} \phi} \alpha \\
i \beta e^{-r_{-} \phi} & e^{-i r_{+} \phi} \sqrt{1-\alpha \beta}
\end{array}\right)
$$

and take $\phi$ to be a periodic variable with period $2 \pi$. The coordinates $\alpha, \beta$ have to satisfy $\alpha \beta \leqslant 1$.

The metric of Eq. (25) is of the form

$$
d s^{2}=\left[r_{+}^{2}-\alpha \beta\left(r_{+}^{2}+r_{-}^{2}\right)\right] d \phi^{2}+\ldots
$$

from which we see that in the region $\alpha \beta>r_{+}^{2}\left(r_{+}^{2}+r_{-}^{2}\right)^{-1}$ the circle parametrized by $\phi$ becomes timelike. Therefore, we should remove this unphysical region.

The region $0 \leqslant \alpha \beta \leqslant r_{+}^{2}\left(r_{+}^{2}+r_{-}^{2}\right)^{-1}$ with $\alpha, \beta>0$ can be parametrized as

$$
\begin{aligned}
\left(\begin{array}{cc}
u & i \alpha \\
i \beta & \bar{u}
\end{array}\right) & \\
= & \frac{1}{\sqrt{r_{+}^{2}+r_{-}^{2}}} \\
& \times\left(\begin{array}{l}
e^{i\left(r_{+} \phi+r_{-} t\right)} \sqrt{r^{2}+r_{-}^{2}} i e^{r_{-} \phi-r_{+} t} \sqrt{r_{+}^{2}-r^{2}} \\
i e^{-r_{-} \phi+r_{+} t} \sqrt{r_{+}^{2}-r^{2}} e^{-i\left(r_{+} \phi+r_{-} t\right)} \sqrt{r^{2}+r_{-}^{2}}
\end{array}\right) .
\end{aligned}
$$

The Kerr-dS metric derived from this reads

$$
\begin{aligned}
d s^{2}= & -\frac{\left(r^{2}+r_{-}^{2}\right)\left(r_{+}^{2}-r^{2}\right)}{r^{2}} d t^{2} \\
& +\frac{r^{2}}{\left(r^{2}+r_{-}^{2}\right)\left(r_{+}^{2}-r^{2}\right)} d r^{2} \\
& +r^{2}\left(d \phi+\frac{r_{+} r_{-}}{r^{2}} d t\right)^{2}
\end{aligned}
$$

which looks very similar to that of the Bañados-TeitelboimZanelli (BTZ) black hole [37] of 3D gravity with $\Lambda<0$. There is a horizon at $r=r_{+}$of circumference $2 \pi r_{+}$. The metric can be continued to $r^{2}>r_{+}^{2}$, where the roles of $t$ and $r$ are interchanged, so that the metric looks like

$$
\begin{aligned}
d s^{2}= & -\frac{r^{2}}{\left(r^{2}+r_{-}^{2}\right)\left(r^{2}-r_{+}^{2}\right)} d r^{2}+r^{2}\left(d \phi+\frac{r_{+} r_{-}}{r^{2}} d t\right)^{2} \\
& +\frac{\left(r^{2}+r_{-}^{2}\right)\left(r^{2}-r_{+}^{2}\right)}{r^{2}} d t^{2}
\end{aligned}
$$

At large $|r|$ this resembles the region close to $\mathcal{I}^{ \pm}$in the Penrose diagram of $\mathrm{dS}_{3}$. Altogether, the Penrose diagram of the Kerr-de Sitter space is similar to the Penrose diagram of de Sitter space, with static patches of the form (28), and two regions near past and future infinity. Near $\mathcal{I}^{-}$the metric (28) becomes

$$
d s^{2} \sim-\frac{d r^{2}}{r^{2}}+r^{2}\left(d \phi^{2}+d t^{2}\right)
$$

and the spacelike slices are cylinders. In contrast to the case of $\mathrm{dS}_{3}$, the points at $t \rightarrow \pm \infty$ of the cylinder are not part of spacetime. The topology of Kerr-de Sitter space is $R^{2} \times S^{1}$, whereas the topology of global de Sitter space is $R \times S^{2}$.

The metric (28) with $r_{-}=0$

$$
d s^{2}=-\left(r_{+}^{2}-r^{2}\right) d t^{2}+\frac{d r^{2}}{\left(r_{+}^{2}-r^{2}\right)}+r^{2} d \phi^{2}
$$

is a conical defect, with deficit angle $2 \pi\left(1-r_{+}\right)$, describing a world with a positive cosmological constant and a pointlike massive observer. When $r_{+}=1$ we reproduce global $\mathrm{dS}_{3}$. As in $\mathrm{AdS}_{3}$, it is interesting to ask whether the spacetimes with $r_{+}>1$ (in effect, "conical excesses") make sense. In $\mathrm{AdS}_{3}$, the AdS/CFT correspondence tells us such spaces should be unphysical, so we might expect that the situation in $\mathrm{dS}_{3}$ is similar.

Another interesting limit is $r_{+} \rightarrow 0$, where the deficit angle becomes $2 \pi$. In this limit, the parametrization we used so far is inadequate. A convenient alternative is

$$
\left(\begin{array}{cc}
u & i \alpha \\
i \beta & \bar{u}
\end{array}\right)=\left(\begin{array}{cc}
e^{i \psi} \sqrt{1+t^{2}} & i e^{r_{-} \phi_{t}} \\
i e^{-r_{-} \phi_{t}} & e^{-i \psi} \sqrt{1+t^{2}}
\end{array}\right) .
$$


Here, both $\psi$ and $\phi$ are periodic variables. The metric derived from Eq. (32) reads

$$
d s^{2}=-\frac{1}{1+t^{2}} d t^{2}+\left(1+t^{2}\right) d \phi^{2}+r_{-}^{2} d \psi^{2} .
$$

The geometry represents a torus that is contracted to a circle as $t \rightarrow 0$. The metric for $-\infty<t \leqslant 0$ is a kind of big crunch solution, the metric for $0 \leqslant t<\infty$ is a big bang type solution. The topology in each case is that of a solid two-torus, exactly the same as that of the BTZ black hole.

More general big bang/big crunch solutions exist, with a metric of a form identical to Eq. (29),

$$
\begin{aligned}
d s^{2}= & -\frac{t^{2}}{\left(t^{2}+r_{-}^{2}\right)\left(t^{2}-r_{+}^{2}\right)} d t^{2} \\
& +t^{2}\left(d \phi+\frac{r_{+} r_{-}}{t^{2}} d r\right)^{2}+\frac{\left(t^{2}+r_{-}^{2}\right)\left(t^{2}-r_{+}^{2}\right)}{t^{2}} d r^{2} .
\end{aligned}
$$

[Here $r$ is periodic, and so the solution cannot be continued through a horizon unlike Eq. (29).] It would be interesting to see whether there exists a change of coordinates which takes this metric to a form similar to the static patch of de Sitter space. An important difference is that we now require that both $r$ and $\phi$ are periodic variables. Therefore, the metric cannot be extended to $-r_{+}<t<r_{+}$. For $t \leqslant-r_{+}$the metric (34) is again some kind of big crunch solution, with a torus contracting to a circle, whereas for $t \geqslant r_{+}$it is a big bang like solution.

The metric for the Kerr-de Sitter solution can be conveniently rewritten as

$$
\begin{aligned}
d s^{2}= & -\left(8 G m-\frac{r^{2}}{l^{2}}+\frac{(8 G J)^{2}}{4 r^{2}}\right) d t^{2} \\
& +\left(8 G m-\frac{r^{2}}{l^{2}}+\frac{(8 G J)^{2}}{4 r^{2}}\right)^{-1} d r^{2} \\
& +r^{2}\left(-\frac{8 G J}{2 r^{2}} d t+d \phi\right)^{2} .
\end{aligned}
$$

\section{B. Mass and angular momentum of Kerr-dS spacetimes}

We begin by studying the Kerr-dS metric with $J=0$. These are the conical defect spacetimes appearing in Eq. (31), approaching empty de Sitter when $8 G m=1$. For these spaces, there is a basic subtlety in evaluating the boundary stress tensor of $\mathrm{dS}_{3}$ that enters the candidate mass formula (18). In the region $r<l \sqrt{8 G m}$, equal $t$ surfaces of Eq. (35) approach the cosmological horizon (rather than $\mathcal{I}^{ \pm}$at early and late times). By contrast, when $r>l \sqrt{8 G m}, \quad r$ becomes timelike while $t$ is a spatial direction, and large $r$ surfaces approach $\mathcal{I}^{ \pm}$. Since we propose to define the stress tensor and mass at $\mathcal{I}^{ \pm}$, we will evaluate these quantities on surfaces of large fixed $r$ which therefore lie outside the cosmological region accessible to the observer creating the conical defect in Eq. (31).

An equal time surface of the conical defect spacetimes outside the cosmological horizon $(r>l \sqrt{8 G m})$ has a metric

$$
d s^{2}=\left(\frac{r^{2}}{l^{2}}-8 G m\right) d t^{2}+r^{2} d \theta^{2} .
$$

We can evaluate the stress tensor (12) on this surface and compute the mass (18); each step is almost identical to the analogous AdS computations [30]. As $r \rightarrow \infty$ (recall $r$ is timelike now) we find

$$
M=\frac{1}{8 \pi G} \oint d \theta \frac{8 G m}{2}=\frac{8 G m}{8 G}=m .
$$

Setting $8 G m=1$ we find that $\mathrm{dS}_{3}$ is assigned a mass of $1 / 8 G$

Surprisingly, we are finding that the conical defects have lower mass than pure de Sitter space. We might interpret this as follows. Even if the matter making up the defects itself has positive energy, the binding energy to the gravitational background can decrease the total energy. In particular, a conical defect "swallows up" a part of the spacetime and thereby appears to reduce the net amount of energy stored in the cosmological constant.

The computation of the Brown-York boundary stress tensor for the general Kerr-de Sitter spacetime parallels the analogous computation for the BTZ black hole [30]. Specifically, if

$$
d s^{2}=-\frac{l^{2}}{r^{2}} d r^{2}+\frac{r^{2}}{l^{2}}\left(d \tau^{2}+d x^{2}\right)+\delta g_{M N} d x^{M} d x^{N}
$$

one finds that the mass and the angular momentum are given by the following expressions:

$$
M=\frac{l}{8 \pi G} \int d x\left[\frac{r^{4}}{2 l^{5}} \delta g_{r r}+\frac{1}{l} \delta g_{x x}-\frac{r}{2 l} \partial_{r} \delta g_{x x}\right]
$$

and

$$
P_{x}=-\frac{l}{8 \pi G} \int d x\left[\frac{1}{l} \delta g_{\tau x}-\frac{r}{2 l} \partial_{r} \delta g_{\tau x}\right]
$$

where in the case of the Kerr-de Sitter solution

$$
\delta g_{r r}=\frac{8 G m l^{4}}{r^{4}}, \quad \delta g_{\tau \tau}=8 G m, \quad \delta g_{\tau \phi}=-4 G J
$$

with $x=l \phi$ and $\phi \in[0,2 \pi]$. Thus the final result is

$$
M=m, \quad P_{\phi}=J .
$$

As a cross-check, note that we recover the mass $M=1 / 8 G$ of de Sitter space if we set $J=0$ and $m=1 / 8 G$.

Our computation of the mass and angular momentum of the Kerr-dS spaces is strongly reminiscent of the techniques 
of Euclidean conformal field theory. The massive spinning defect intersects a point on the Euclidean surface at $\mathcal{I}^{+}$ which is inside the cosmological horizon and excised from the exterior region. We find the charges carried by the defect performing a contour integral around this excised point. This computation mimics the usual CFT procedure of inserting an operator at the origin around which we integrate to compute charges.

\section{CONFORMAL SYMMETRIES AND ENTROPY OF $\mathrm{dS}_{3}$}

We will now employ the boundary stress tensor developed in the previous section in the study of the symmetry group of asymptotically $\mathrm{dS}_{3}$ spaces. We will first study the $S L(2, C)$ isometry group and then the group of asymptotic conformal symmetries.

\section{A. Isometries}

We will set the de Sitter scale $l$ to 1 , restoring it as needed by dimensional analysis. In Eqs. (21),(22) we indicated how $\mathrm{dS}_{3}$ can be represented as the group manifold $S L(2, C) / S L(2, R)$. We can represent each of the three metrics for de Sitter space that we discussed earlier (inflating, global and static) in terms of the matrix $M$. Global coordinates for $\mathrm{dS}_{3}$ (3) correspond to parametrizing $M$ as

$$
M=\left(\begin{array}{cc}
\cosh t \sin \theta e^{i \phi} & i(\sinh t+\cosh t \cos \theta) \\
i(-\sinh t+\cosh t \cos \theta) & \cosh t \sin \theta e^{-i \phi}
\end{array}\right),
$$

with induced metric $d s^{2}=-d t^{2}+\cosh ^{2} t\left(d \theta^{2}+\sin ^{2} \theta d \phi^{2}\right)$. The big bang inflationary patch (4) corresponds to $\alpha>0$. This is parametrized as

$$
\begin{aligned}
M & =\left(\begin{array}{cc}
e^{t} z & i e^{t} \\
i\left(e^{-t}-e^{t} z \bar{z}\right) & e^{t} \bar{z}
\end{array}\right) \\
& \Rightarrow d s^{2} \\
& =-d t^{2}+e^{2 t} d z d \bar{z} .
\end{aligned}
$$

Similarly, one can parametrize the big bang/big crunch and inflationary patches corresponding to $\alpha<0, \beta>0$, and $\beta$ $<0$. A static patch (5) is covered by $\alpha>0, \beta>0$. The matrix $M$ is parametrized as

$$
\begin{aligned}
M & =\left(\begin{array}{cc}
e^{i \phi} r & i e^{t} \sqrt{1-r^{2}} \\
i e^{-t} \sqrt{1-r^{2}} & e^{-i \phi} r
\end{array}\right) \\
& \Rightarrow d s^{2} \\
& =-\left(1-r^{2}\right) d t^{2}+\frac{1}{1-r^{2}} d r^{2}+r^{2} d \phi^{2} .
\end{aligned}
$$

\section{$S L(2, C)$ action}

One convenient feature of the parametrization in terms of the matrix $M$ is that it is easy to describe the action of the
$S L(2, C)$ isometry group on the coordinates via Eq. (23). From this we get the relevant Virasoro action in the different patches. Notice that the actual real generators are of the form $\alpha L_{i}+\bar{\alpha} \bar{L}_{i}$, where $\bar{L}_{i}$ is the complex conjugate of $L_{i}$. For the global patch we get

$$
\begin{gathered}
L_{-1}=\frac{i}{2} e^{-i \phi}\left(\cot \theta+\frac{\tanh t}{\sin \theta}\right) \partial_{\phi}-\frac{1}{2} e^{-i \phi} \sin \theta \partial_{\theta} \\
-\frac{1}{2} e^{-i \phi}(\cosh t+\cos \theta \sinh t) \partial_{t} \\
L_{0}=\frac{i}{2} \partial_{\phi}+\frac{1}{2} \cos \theta \partial_{\theta}-\frac{1}{2} \sin \theta \tanh t \partial_{t} \\
L_{1}=-\frac{i}{2} e^{i \phi}\left(\cot \theta-\frac{\tanh t}{\sin \theta}\right) \partial_{\phi}+\frac{1}{2} e^{i \phi} \sin \theta \partial_{\theta}+\frac{1}{2} e^{i \phi} \\
\times(-\cosh t+\cos \theta \sinh t) \partial_{t}
\end{gathered}
$$

In the inflationary patch we obtain

$$
\begin{gathered}
L_{-1}=-\partial_{z} \\
L_{0}=\frac{1}{2} \partial_{t}-z \partial z \\
L_{1}=z \partial_{t}-z^{2} \partial_{z}-e^{-2 t} \partial_{\bar{z}}
\end{gathered}
$$

and in the static patch

$$
\begin{aligned}
L_{-1}= & -\frac{1}{2 r}\left(1-r^{2}\right)^{1 / 2} e^{t-i \phi}\left(\partial_{t}-2 r \partial_{r}\right) \\
& +\frac{i r}{2}\left(1-r^{2}\right)^{-1 / 2} e^{t-i \phi} \partial_{\phi} \\
L_{0}= & \frac{1}{2} \partial_{t}+\frac{i}{2} \partial_{\phi} \\
L_{1}= & \frac{1}{2 r}\left(1-r^{2}\right)^{1 / 2} e^{-t+i \phi}\left(\partial_{t}+2 r \partial_{r}\right) \\
& -\frac{i r}{2}\left(1-r^{2}\right)^{-1 / 2} e^{-t+i \phi} \partial_{\phi} .
\end{aligned}
$$

In particular, we see that in the static patch, $L_{0}+\bar{L}_{0}=\partial_{t}$.

\section{B. Asymptotic conformal symmetry}

Brown and Henneaux [38] specified boundary conditions for asymptotically $\mathrm{AdS}_{3}$ spaces that admitted a well defined algebra of diffeomorphisms. These continue to boundary conditions at future or past infinity of $\mathrm{dS}_{3}$, defining an asymptotically de Sitter geometry. Working in the inflating coordinate system (4) we obtain that an asymptotically $\mathrm{dS}_{3}$ space has a metric that satisfies [10] 


$$
\begin{gathered}
g_{+-}=-\frac{e^{2 \tau / l}}{2}+O(1), \quad g_{++}, g_{--}=O(1), \\
g_{\tau \tau}=-1+O\left(e^{-2 \tau / l}\right), \quad g_{+r}, g_{-r}=O\left(e^{-3 \tau / l}\right) .
\end{gathered}
$$

The diffeomorphisms that respect these conditions are given in terms of functions $\xi^{+}\left(z^{+}\right)$and $\xi^{-}\left(z^{-}\right)$, and take the asymptotic form [10]

$$
\begin{gathered}
z^{+} \rightarrow z^{+}-\xi^{+}-\frac{l^{2}}{2} e^{-2 \tau / l} \partial_{-}^{2} \xi^{-} \\
z^{-} \rightarrow z^{-}-\xi^{-}-\frac{l^{2}}{2} e^{-2 \tau / l} \partial_{+}^{2} \xi^{+} \\
e^{\tau / l} \rightarrow e^{\tau / l}+\frac{e^{\tau / l}}{2}\left(\partial_{+} \xi^{+}+\partial_{-} \xi^{-}\right) .
\end{gathered}
$$

To understand the meaning of these diffeomorphisms, consider the metric $\gamma_{\mu \nu}$ induced on an equal time surface of large $\tau$ in Eq. (4). To leading order in $\tau$, Eqs. (56) and (57) produce a conformal transformation of this metric. The resulting conformal factor in $\gamma_{\mu \nu}$ is undone by the effective Weyl transformation induced by Eq. (58), leaving the leading asymptotic metric unchanged [10]:

$$
\begin{aligned}
d s^{2} \rightarrow & -d \tau^{2}+e^{2 \tau / l} d z^{+} d z^{-}+\frac{l^{2}}{2}\left(\partial_{+}^{3} \xi^{+}\right)\left(d z^{+}\right)^{2} \\
& +\frac{l^{2}}{2}\left(\partial_{-}^{3} \xi^{-}\right)\left(d z^{-}\right)^{2} .
\end{aligned}
$$

We learn from this that the asymptotic symmetry group of $\mathrm{dS}_{3}$, subject to the boundary conditions (55), is the two dimensional Euclidean conformal group, which contains the isometries discussed above as a subgroup. Indeed, the detailed analysis of Brown and Henneaux [38] can be formally analytically continued to arrive at a Virasoro symmetry algebra, but we will not present the details here. Clearly, a similar analysis can be carried out to show that there is a conformal group of asymptotic symmetries at past infinity also.

In summary, the asymptotic symmetry group of de Sitter space is the conformal group. Interestingly, time translation in the inflating patch of de Sitter space is related to the dilatation generator of the conformal group. Explicitly, the isometry generator $(50)$ in the static patch shows that a dilatation of the boundary can be undone by a time translation. It is also illuminating to rewrite the isometry generators (49)(51) using the conformal time introduced in Eq. (4). They become:

$$
\begin{gathered}
L_{-1}=-\partial_{z}, \quad L_{0}=-\frac{\eta}{2} \partial_{\eta}-z \partial_{z}, \\
L_{1}=-z \eta \partial_{\eta}-z^{2} \partial_{z}-\eta^{2} \partial_{z}^{-} .
\end{gathered}
$$

It is then clear that as $\eta \rightarrow 0$, reaching the boundary of de Sitter space, the isometries reduce to standard conformal transformations of the plane, while $L_{0}$ and the conjugate $\bar{L}_{0}$ generate dilatations of the boundary. As we will see later, if there is a holographic dual to de Sitter space, this leads to a correspondence between the renormalization group of the dual and time translation in de Sitter space.

We will compute the central charge of the asymptotic conformal symmetry using the methods of [30]. We have shown in this section that there is an asymptotic group of conformal symmetries. However, as we will see, the action (7) is not left invariant by these conformal transformations; there is an anomaly produced by the procedure of cutting off the space to regulate divergences and then removing the cutoff. [This anomaly is intimately connected to the logarithmic divergences discussed below Eq. (9). Such divergences and the resulting conformal anomlies were discussed for asymptotically AdS spaces in [29,32].] The stress tensor (12) computes the change in the action to variations of the boundary metric. Hence we can measure the central charge of the conformal symmetry group of $\mathrm{dS}_{3}$ by computing the anomalous transformation of this stress tensor under the diffeomorphisms (56) - (58), which produce the transformed metric (59). We showed in Eq. (14) that the boundary stress tensor of the inflating patch vanishes. After the diffeomorphisms (56),(57), the boundary stress tensor becomes

$$
T_{++}=-\frac{l}{16 \pi G} \partial_{+}^{3} \xi+, T_{--}=-\frac{l}{16 \pi G} \partial_{-}^{3} \xi-.
$$

Using the standard formulas for the anomalous transformation of the stress tensor in a two dimensional conformal theory, we read off the central charge $c=-3 l / 2 G$, which has the same formal dependence on the cosmological length scale as the central charge of $\mathrm{AdS}_{3}$. The negative sign of $c$ is not problematic here since we are dealing with a classical stress tensor. If, as metioned before, we had chosen to define a stress tensor with the opposite overall sign as $(-2 / \sqrt{h}) \delta I / \delta h_{\mu \nu}$, the resulting object would of course have a positive central charge under conformal transformations. The same result for the central charge is obtained by examining the trace of the stress tensor in global coordinates as $t \rightarrow \infty$ (15). Remembering that $T=-(c / 24 \pi) R$, where $R$ is the scalar curvature of the spherical boundary, we again find $c=-3 l / 2 G$.

The analysis outlined above can be repeated in four and five dimensions. We can compute the Brown-York stress tensor on the spacetime boundary, and the anomalous variation of this tensor for five dimensional de Sitter space will yield a central charge. However, the absence of such an anomaly for four dimensional de Sitter space prevents use of this tool in that case. All computations proceed in analogy to those in [30].

\section{Brown-York, Cardy and Bekenstein-Hawking}

The Kerr-dS spacetimes (35) have cosmological horizons giving rise to a Bekenstein-Hawking entropy [39-41]

$$
S_{B H}^{K-d S}=\frac{\sqrt{2} \pi l}{4 G} \sqrt{(8 G m)+\sqrt{(8 G m)^{2}+\frac{(8 G J)^{2}}{l^{2}}}} .
$$


In particular when $J=0$, the horizon is at $r=l \sqrt{8 G m}$ and the entropy is $S=\pi l \sqrt{8 G m} / 2 G$. As illustrated in the previous sections (also see $[33,9,10,15]$ ) there is an asymptotic conformal algebra in $\mathrm{dS}_{3}$ in detailed analogy with the $\mathrm{AdS}_{3}$ symmetry algebra uncovered by Brown and Henneaux [38]. Therefore, if there is a holographic dual to de Sitter space, we might expect it to be a Euclidean conformal field theory $[9,10]$. Alternatively, the dual theory might be in fact a Lorentzian conformal field theory with a Euclidean signature Virasoro algebra. In addition, Strominger has emphasized that the results of Brown and Henneaux can be understood as saying that states of any well defined quantum gravity on $\mathrm{AdS}_{3}$ transform in representations of the conformal group, and that the same applies to $\mathrm{dS}_{3}$.

If there is a duality between de Sitter space and a CFT it is not yet on a solid footing - we neither know how to realize de Sitter space in string theory, nor how the gravitational data could be related to a dual. However, it might be natural to suppose that in analogy with AdS, the dS stress tensor is related to the stress tensor of a dual. Indeed, the actual definition of spacetime conserved charges in Eqs. (17),(18),(19) is in formal analogy to the standard definition of conserved charges in a Euclidean CFT. In effect our treatment excises a point on $\mathcal{I}^{+}$where the static patch meets future or past infinity and the contour integral in Eq. (17) is carried out around this point. We might imagine that from the perspective of a dual theory the operator responsible for creating the spacetime state is placed at this excised point and our formula computes the charges of the state after mapping the plane to the cylinder.

However, in making such an identification there are several subtle issues. First of all, there are various possible sign flips that may be relevant. For example, if we want to follow the radial quantization analogy given above, the Euclidean time coordinate obtained by continuation out of the static patch flows in the wrong direction at $\mathcal{I}^{+}$. (After mapping to the plane it flows towards the radial origin where the operator on $\mathcal{I}^{+}$would be inserted rather than towards radial infinity.) For similar reasons, CFT stress tensors on $\mathcal{I}^{+}$and $\mathcal{I}^{-}$ that describe the same space might be related to our dS stress tensor with opposite signs. Issues of this kind can be solved properly if an actual technical definition of a dS/CFT correspondence is devised. Here we content ourselves with the following tempting numerological observation.

The considerations above, coupled with Strominger's well-known observations regarding the entropy of the BTZ black hole [42], suggest that the entropy of Kerr-dS spaces could be explained by applying a Cardy-like formula to a CFT with left and right energy levels measured by the eigenvalues of the $L_{0}$ and $\bar{L}_{0}$ conformal generators in static coordinates, as defined in Eq. (53). From their definition, we see that these $L_{0}$ and $\bar{L}_{0}$ eigenvalues are related to the mass (18) and angular momentum (19) by

$$
L_{0}+\bar{L}_{0}=l m, \quad L_{0}-\bar{L}_{0}=i J
$$

Formally, this gives

$$
L_{0}=\frac{1}{2}(m l+i J), \quad \bar{L}_{0}=\frac{1}{2}(m l-i J) .
$$

(Also see [10] for details relating the Brown-York stress tensor to the Virasoro generators.) When $J=0$, naive application of a Cardy-like formula for the asymptotic density of states of a unitary CFT,

$$
S_{C}=2 \pi \sqrt{\frac{|c| L_{0}}{6}}+2 \pi \sqrt{\frac{|c| \bar{L}_{0}}{6}}
$$

gives the entropy

$$
S=\pi l \sqrt{\frac{8 G m}{2 G}}
$$

in exact agreement with the entropy of the conical defects (31) (also see [14]). ${ }^{6}$

Now observe that the spinning Kerr-dS spacetimes give rise to complex eigenvalues for $L_{0}$ and $\bar{L}_{0}$. A CFT with these left and right energy levels therefore cannot be unitary. Nevertheless, we naively apply a Cardy-like formula (65) and find that

$$
\begin{gathered}
S=2 \pi \sqrt{\frac{|c|(m l+i J)}{12}+2 \pi \sqrt{\frac{|c|(m l-i J)}{12}}} \\
=\frac{\sqrt{2} \pi l}{4 G} \sqrt{(8 G m)+\sqrt{(8 G m)^{2}+\frac{(8 G J)^{2}}{l^{2}}}} .
\end{gathered}
$$

The complex $L_{0}$ eigenvalues suggest that the Cardy formula cannot be valid, since it does not generally apply to nonunitary theories, but we have nevertheless exactly reproduced the Kerr-dS entropy (62).

In fact, there is a further subtlety. The complete Cardy formula for the asymptotic density of states of a unitary $2 \mathrm{D}$ CFT is:

$$
S_{C}=2 \pi \sqrt{\frac{c}{6}\left(L_{0}-c / 24\right)}+2 \pi \sqrt{\frac{c}{6}\left(\bar{L}_{0}-c / 24\right)}
$$

and we might expect large corrections when $L_{0}, \bar{L}_{0} \sim c / 24$. For $\mathrm{dS}_{3}, \quad M=1 / 8 G$ and so $L_{0}=\bar{L}_{0}=1 / 16 G=|c| / 24$ and so it is surprising that dropping the $|c| / 24$ shift still gives the "right" answer for the entropy. The important point is that the entropy of $\mathrm{dS}_{3}$ (and of the conical defects) scales linearly

\footnotetext{
${ }^{6}$ We have placed absolute value signs around $c$ because in our definition of the spacetime stress tensor $c<0$. As we mentioned above there are several subtle issues in relating the signs of the spacetime stress tensor to possible duals and one hopes that these issues could be resolved given a technical definition of a fullfledged duality. Regardless, the numerological observation made here remains interesting.
} 
with the central charge. We have in fact found that in generic CFTs with a central charge $c$, when $L_{0} \sim c / 24$, the degeneracy of states behaves as

$$
d \sim e^{\lambda c}
$$

with a non-universal coefficient $\lambda$. Also, if the CFT dual to $\mathrm{dS}_{3}$ is related to the D1-D5 CFT which was dual to $\mathrm{AdS}_{3}$, one might be able to use $U$ duality to show that the formula (65) applies even when $L_{0} \sim c / 24$.

Actually, in the above discussion we have been somewhat imprecise regarding the Hermiticity conditions and reality conditions on $L_{k}$ and $\bar{L}_{k}$. As one can see from Eqs. (52)(54), the $L_{k}$ satisfy the Hermiticity condition $L_{k}^{\dagger}=-\bar{L}_{k}$. On the other hand, the eigenvalues given in Eq. (64) are not compatible with this Hermiticity condition. The reason is that conventionally, the energy or mass is the eigenvalue associated to the operator $-i \partial_{t}$, while the mass is computed from the Brown-York tensor using the $\partial_{t}$ Killing vector. In our case, the operator $-i \partial_{t}$ is $-i\left(L_{0}+\bar{L}_{0}\right)$, as is clear from Eqs. (52)-(54). Therefore, we could have identified $l m$ with $-i\left(L_{0}+\bar{L}_{0}\right)$, and $i J$ with $-i\left(L_{0}-\bar{L}_{0}\right)$, which gives $L_{0}$ $=i / 2(m l+i J)$ and $\bar{L}_{0}=i / 2(m l-i J)$. At the same time, the modes of the stress tensor as we defined it would not be identified with $L_{k}$, but rather with $i L_{k}$. This implies that the central charge should have been equal to $-3 i l / 2 G$. Putting these values for $L_{0}, \bar{L}_{0}$ and $c$ into the Cardy formula reproduces the Kerr-dS entropy; they are also identical to the values obtained from a Chern-Simons theory analysis by Park [13]. The central lesson to be learned here is that while relating a Lorentzian bulk to a Euclidean boundary there are factors of $i$ and unusual reality conditions which will be important to understand for the definition of a possible dS/CFT correspondence.

It is interesting to note that similar conclusions can be reached by naively continuing the AdS results in [42] to dS by complexifying the scale: $l \rightarrow i l$. The central charge and $L_{0}$ eigenvalues become imaginary, but conspire to correctly give the entropies of the de Sitter conical defects in a naive application of the Cardy formula.

Finally, the expression for the Brown-York mass of a conical defect allows us to compute the Hawking temperature from the first law of thermodynamics,

$$
d E=T d S,
$$

and the fact that $E=\gamma^{2} / 8 G$, while the entropy is $S$ $=\gamma \pi l / 2 G$. (We have written $\gamma^{2}=8 G m$.) By considering variations over $\gamma$ we deduce that

$$
T_{H}=\frac{d E}{\mathrm{dS}}=\frac{\gamma}{2 \pi l}
$$

as it should be $[43,40,41]$. In the limit when $\gamma=1$ we obtain the correct expression for the Hawking temperature of de Sitter space.

Banks has emphasized that the finite entropy of de Sitter space leads us to expect that quantum gravity in a universe with a positive cosmological constant has a finite number of degrees of freedom $[44,45]$. It is worth examining how this might be reconciled with the proposition that a Euclidean conformal field theory may be dual to de Sitter space, since any local field theory has an infinite number of degrees of freedom. The picture emerged above is that de Sitter space should be described by an ensemble of states with $L_{0}=\bar{L}_{0}$ $=|c| / 24$. The finite degeneracy of such states would account for de Sitter entropy. (This is reminiscent of [46].) Likewise, states of lower $L_{0}$ contribute to ensembles that describe the classical conical defects. In this picture it is natural that the conical defects and Kerr-dS spaces have masses smaller than that of $\mathrm{dS}_{3}$.

But what about states with the real part of $L_{0}>|c| / 24$ ? These correspond to spacetimes with mass bigger than that of $\mathrm{dS}_{3}$. Consider such a universe, which is asymptotically $\mathrm{dS}_{3}$ at early times and in which the mass formula (18) at $\mathcal{I}^{-}$ measures a mass $M>1 / 8 G$. It is likely that such a space evolves to a singularity and is not asymptotically de Sitterlike in the future. Hence, most states, having $\operatorname{Re}\left(L_{0}\right)$ $>|c| / 24$, would not lead to de Sitter-like evolution and therefore the specification of de Sitter boundary conditions would restrict the dual theory to a finite number of states. In turn this implies that the conical parameter $8 G m \leqslant 1$.

\section{HIGHER DIMENSIONAL de SITTER SPACE AND A MASS BOUND}

We can test the picture of de Sitter entropy that emerged in the previous section by examining what our mass formula says about black holes in higher dimensions. When $d+1$ $>3$ the action (1) admits Schwarzschild-dS black hole solutions of the form

$$
\begin{gathered}
d s^{2}=-V(r) d t^{2}+V(r)^{-1} d r^{2}+r^{2} d \Omega_{d-2}, \\
V(r)=1-\frac{2 G m}{r^{d-2}}-\frac{2 \Lambda r^{2}}{d(d-1)}=1-\frac{2 G m}{r^{d-2}}-\frac{r^{2}}{l^{2}} .
\end{gathered}
$$

This space has horizons at locations where $V(r)=0$.

When $m=0$ we recover static coordinates for empty de Sitter space with a single cosmological horizon at $r=l$ and an entropy $S=l^{d-2} V_{d-2} / 4 G$ where $V_{d-2}$ is the volume of the unit $(d-2)$ sphere. As $m$ increases, a black hole horizon appears, increasing in size with $m$. Simultaneously the cosmological horizon shrinks in size, pulled inwards by the gravitational attraction of the black hole. As a result there is the largest black hole, the Nariai solution, which occurs when

$$
m=m_{N}=\left(\frac{1}{d G}\right)\left[\frac{(d-1)(d-2)}{2 \Lambda}\right]^{(d-2) / 2} .
$$

For this critical choice of mass, the black hole and cosmological horizons coincide at a radius $r^{2}=(d-1)(d-2) / 2 \Lambda$ $=l^{2}(d-2) / d$, giving rise to a Bekenstein-Hawking entropy of $S=l^{d-2} V_{d-2}(1-2 / d)^{(d-2) / 2} / 4 G$. Spaces with $m>m_{N}$ have unacceptable naked singularities. The Nariai solution can also be reparametrized as (e.g., see [53]) 


$$
\begin{array}{r}
d s^{2}=-W(r) d t^{2}+W(r)^{-1} d r^{2} \\
+\frac{(d-1)(d-2)}{2 \Lambda} d \Omega_{d-2}, \\
W(r)=1-\frac{2 \Lambda r^{2}}{(d-1)}=1-\frac{d r^{2}}{l^{2}} .
\end{array}
$$

As we noted above, the Nariai solution has a smaller entropy than that of de Sitter space. Indeed, the sum of the entropies of black hole horizons, cosmological horizons and matter fields cannot exceed the entropy of de Sitter space, given suitable energy conditions on the matter [20].

We can compute the masses of the black holes (73) using Eq. (18). In four dimensions we find that

$$
M_{4}=-m \text {. }
$$

So $\mathrm{dS}_{4}$ has a vanishing mass according to our formula while the Nariai black hole has $M_{4}=-(1 / 3 G \sqrt{\Lambda})$. In five dimensions the mass formula becomes

$$
M_{5}=\frac{3 \pi l^{2}}{32 G}-\frac{3 \pi m}{4}
$$

(Note that this result differs in sign from [15].) So the mass of $\mathrm{dS}_{5}$ is $2 \pi l^{2} / 32 G$, and, pleasantly, the mass of the $5 \mathrm{D}$ Nariai black hole is 0 . This is parallel to the three dimensional case where the biggest conical defect had a mass of zero.

If there is a CFT dual to $\mathrm{dS}_{4}$ and $\mathrm{dS}_{5}$, defined in the style of the AdS/CFT correspondence [11,12,6-10], the masses that we have computed translate into the energies of a dual Euclidean conformal field theory. Generically such theories have entropies that increase with energy. Therefore, if they are also to reproduce the decreasing entropy of larger black holes, these spaces should map into ensembles of decreasing energy. Our results (78), (79) have precisely this property. In this regard, the fact that the 4D masses are negative need not be worrisome because there can easily be a shift in the formula relating energies to entropy.

It is also interesting that the numerical mass of de Sitter space in all three examples that we have studied is the same (up to a sign) as the numerical mass of $\mathrm{AdS}$ spaces in the same dimension [30]. This may be an indication that if duals to de Sitter space exist, they may be related to the duals already known for AdS spaces. Indeed, in the Chern-Simons formulation of $2+1$ gravity, intriguing relations are known between the theories with positive and negative cosmological constants. Classical Euclidean $\mathrm{AdS}_{3}$ gravity and Lorentzian $\mathrm{dS}_{3}$ are Chern-Simons theories of the same group, but are endowed with different Hilbert space structures [47]. (We hope to return to this in a later publication.)

The total entropy of black hole and cosmological horizons can be computed in gravity for general $m$. It would be interesting to test whether a Cardy-Verlinde-like expression for the asymptotic density of states of a higher dimensional CFT [48] could reproduce this entropy.

\section{A bound on de Sitter masses}

Bousso has shown that, under suitable positive energy conditions on matter fields, the entropy of de Sitter space is an upper bound on the total entropy that can be stored in a space with a positive cosmological constant [20,49]. Above we have shown that our measure of mass increases monotonically as the entropy increases, and argued that it would be natural to map this quantity into the energy of states in a dual field theory. The entropy of de Sitter space is then understood as the degeneracy of such states. Now, consider a space which is asymptotically de Sitter-like in the past, but which has a mass measured by Eq. (18) exceeding the mass of de Sitter space. From a field theory perspective an ensemble with this larger energy would have a larger entropy. Therefore, in view of the de Sitter entropy bound, we conjecture the following: Any asymptotically de Sitter space whose mass as defined in Eq. (18) exceeds that of de Sitter space contains a cosmological singularity. Note that spaces with masses less than or equal to de Sitter space may still be singular for other reasons.

A potential counterexample to our conjecture is provided by the negative-mass Schwarzschild-de Sitter spacetime, in which the timelike singularity always remains within a single cosmological region. However, this space is nakedly singular and the Cauchy problem is actually not well defined-hence it is unclear whether the space can be admitted into consideration. In addition, small fluctuations are likely to lead to evolution in which the singularity propagates along the null cone of the past cosmological horizon cutting off the "lower triangular" region outside the cosmological horizon in a Penrose diagram of de Sitter space. ${ }^{8}$

\section{RG FLOW VERSUS COSMOLOGICAL EVOLUTION}

Finally, we would like to consider the meaning of the holographic [50] UV/IR relation [51] in de Sitter space. Our discussion is motivated by a possible dS/CFT correspondence $[6,8-10]$ in the manner of the AdS/CFT duality $[9,10]$. In analogy with the AdS/CFT correspondence the prescription for the computation of the boundary stress tensor, as presented above, leads quite naturally to the relation between the trace of the stress tensor and the RG equation of a putative dual field theory. In particular, the precise relation between the generators of dilatations on the boundary and the generator of time translations in the bulk, as discussed in Sec. IV B, points to a natural relation between the RG flows of a possible dual boundary theory and the time evolution in the bulk of de Sitter space. This statement is completely analogous to the known relation between the RG transformations and bulk equations of motion in the context of the AdS/CFT correspondence [24-26].

\footnotetext{
${ }^{7}$ We have mentioned several times that we could have chosen to define a de Sitter stress tensor as $(-2 / \sqrt{h}) \delta I / \delta h_{\mu \nu}$, thereby reversing the sign of our mass formula. With this definition, we would be making a positive mass conjecture: all non-singular asymptotically de Sitter spacetimes have mass greater than de Sitter space.

${ }^{8}$ We thank Rob Myers for discussions of this point.
} 
In particular, consider the Wick rotated "kink" solutions of [23] which were found in the context of the AdS/CFT duality. These solutions describe spaces interpolating between two asymptotically $\mathrm{AdS}_{5}$ spaces and are dual to well understood RG flows of supersymmetric 4D field theories. Wick rotating these "kink" spaces produces solutions that correspond to Friedmann-Robertson-Walker (FRW) cosmologies interpolating between two de Sitter vacua. It is well known that RG flows discussed by [23] are characterized by a positive and monotonic $c$-function provided that a weak energy condition is satisfied in the bulk gravitational theory. This holographic $c$-function coincides with the trace anomaly of the dual field theory, and thus can be precisely related to the trace of the boundary stress tensor [26]. In our situation, we can analogously postulate a holographic $c$-function determined by the expression for the trace of the Brown-York tensor and dimensional analysis [26]. The candidate holographic $c$-function is proportional to

$$
c \sim \frac{1}{\left(A^{\prime}\right)^{D-2}}
$$

in the case of a Wick rotated kink solution interpolating between two $D$-dimensional de Sitter spaces, where the metric of the kink solution is

$$
d s^{2}=-d t^{2}+e^{A} \eta_{i j} d x^{i} d x^{j}
$$

Here the prime denotes the $t$ derivative. The asymptotic form of the Wick rotated warp factor is $A=\sqrt{\Lambda} t$. Inserting this expression into the formula for the holographic $c$-function gives the correct scaling of the expected number of degrees of freedom in the asymptotic de Sitter region (essentially determined by the value of the holographic $c$-function at the fixed point) with the cosmological constant $\Lambda$, as implied by the Bekenstein-Hawking entropy formula. ${ }^{9}$

The correspondence between the RG evolution and the bulk cosmological time evolution offers a nice reinterpretation of the monotonicity of our candidate holographic $c$-function from a cosmological point of view. In our scenario, the IR fixed point corresponds to the period of inflation which is driven by a large effective cosmological constant $\Lambda_{\text {inf }}$ - the number of degrees of freedom being proportional to $1 / \Lambda_{\text {inf }}$-in $4 \mathrm{D}$. On the other hand, the UV fixed point corresponds to an asymptotically de Sitter space with a small positive cosmological constant $\Lambda_{o b s}$ (as implied by current observational data). This in turn, according to the relation between the $c$-function and the cosmological constant, corresponds to a large number of degrees of freedom proportional to $1 / \Lambda_{o b s}$. Indeed, this is consistent with a holographic $c$-theorem according to which $c_{\mathrm{UV}}>c_{\mathrm{IR}}$.

In the AdS/CFT correspondence, radial flow of the bulk spacetime solutions corresponded in the dual field theory to RG flow [24-26]. Here we are proposing a relationship between the RG flow of a putative holographic dual to de Sitter

\footnotetext{
${ }^{9}$ These observations were originally made in collaboration with Petr Horava.
}

space and time evolution. In a theory of gravity, the bulk Hamiltonian is zero, and so this is really a map between a holographic RG equation and the Hamiltonian constraint of the bulk gravitation theory, which at the quantum mechanical level becomes the Wheeler-DeWitt equation. (The correspondence between boundary dilatations and bulk time translations is also an example of the spacetime uncertainty relation [52].)

More precisely, following the treatment of holographic $\mathrm{RG}$ flows in asymptotically AdS spaces, we fix the gauge so that the bulk metric can be written as

$$
d s^{2}=-d t^{2}+g_{i j} d x^{i} d x^{j} .
$$

The Hamiltonian constraint reads

$$
\mathcal{H}=0
$$

where in the case of 5D bulk gravity

$$
\mathcal{H}=\left(\pi^{i j} \pi_{i j}-\frac{1}{3} \pi_{i}^{i} \pi_{j}^{j}\right)+\frac{1}{2} \pi_{I} G^{I J} \pi_{J}+\mathcal{L} .
$$

Here $\pi_{i j}$ and $\pi_{I}$ are the canonical momenta conjugate to $g^{i j}$ and $\phi^{I}\left(\phi^{I}\right.$ denotes some background test scalar fields). $\mathcal{L}$ is a local Lagrangian density and $G^{I J}$ denotes the metric on the space of background scalar fields.

As in the context of the AdS/CFT duality [25], the Hamiltonian constraint can be formally rewritten as a CallanSymanzik equation for the dual RG flow

$$
\frac{1}{\sqrt{g}}\left[\frac{1}{3}\left(g^{i j} \frac{\delta S}{\delta g^{i j}}\right)^{2}-\frac{\delta S}{\delta g^{i j}} \frac{\delta S}{\delta g_{i j}}-\frac{1}{2} G^{I J} \frac{\delta S}{\delta \phi^{I}} \frac{\delta S}{\delta \phi^{I}}\right]=\sqrt{g} \mathcal{L},
$$

provided the local 5D action $S$ can be separated into a local and a non-local piece

$$
S(g, \phi)=S_{l o c}(g, \phi)+\Gamma(g, \phi) .
$$

In that case the Hamiltonian constraint can be formally rewritten as a Callan-Symanzik RG equation

$$
\frac{1}{\sqrt{g}}\left(g^{i j} \frac{\delta}{\delta g^{i j}}-\beta^{I} \frac{\delta}{\delta \phi^{I}}\right) \Gamma=H O
$$

where $\mathrm{HO}$ denotes higher derivative terms. Here the "betafunction" is defined (in analogy with the AdS situation) to be $\beta^{I}=\partial_{A} \phi^{I}$ where $A$ denotes the cutoff of the putative dual Euclidean theory.

In Eq. (80) we proposed a holographic $c$-function for asymptotically de Sitter spaces taking a special form. More generally, in analogy with the AdS/CFT correspondence [26], the holographic $c$-function can be related to the extrinsic curvature, or equivalently, to the trace of the Brown-York tensor. In a five dimensional bulk space we would have

$$
c \sim \frac{1}{G \theta^{3}}
$$


Here, as we have seen

$$
\left\langle T_{i}^{i}\right\rangle \sim \theta
$$

up to some terms constructed from local intrinsic curvature invariants of equal time surfaces. The RG equation is given by the conformal Ward identity for the trace of the stress tensor

$$
\left\langle T_{i}^{i}\right\rangle \sim \frac{d \Gamma}{d A}=\beta^{I} \frac{\partial \Gamma}{\partial \phi^{I}} .
$$

The Raychauduri equation then implies the monotonicity of the trace of the Brown-York stress tensor

$$
\frac{d \theta}{d t} \leqslant 0
$$

as long as a form of the weak positive energy condition is satisfied by the background test scalar fields. This in turn guarantees the fundamental monotonicity property we would require of a holographic $c$-function.

Finally, there might be a holographic reinterpretation of the known gravitational instabilities of de Sitter space [53]. One might speculate that the splitting of a de Sitter space into two asymptotically de Sitter spaces [53] could be described by adding non-linear terms in the Wheeler-DeWitt equation. It is tempting to conjecture that some non-linear version of the Wheeler-DeWitt equation can be related to the fully non-linear Wegner-Wilson-Polchinski [54] nonperturbative RG equations. This in turn might imply an interesting revival of the wormhole ideas [55] in the context of a possible dS/CFT correspondence.

\section{DISCUSSION}

In this paper we have computed the Brown-York boundary stress tensor of asymptotically de Sitter spacetimes and used it to define a novel notion of mass and conserved charges. We were motivated to carry out this procedure in order to study the prospects for a duality between quantum gravity on de Sitter space and a Euclidean field theory defined on the spacelike surfaces at $\mathcal{I}^{ \pm}$. The quantities we compute would be the stress tensor and charges of the dual theory, if such a theory were defined for de Sitter space in a manner analogous to the AdS/CFT correspondence $[9,10,8,6,12]$.

Several interesting results have emerged. In all dimensions, we found that classical objects like black holes placed in a world with a positive cosmological constant have masses less than the mass of de Sitter space itself, and we conjecture that all non-singular asymptotically de Sitter spacetimes have mass less than de Sitter space. ${ }^{10}$ These facts make it possible

\footnotetext{
${ }^{10}$ We could have chosen the opposite overall sign for our definition of a stress tensor and mass for de Sitter space in which case we would have a conjecture that all non-singular asymptotically de Sitter spacetimes have masses greater than or equal to de Sitter space.
}

that the entropy of asymptotically de Sitter spacetime could be explained from the density of states of a dual field theory at an energy level equal to the mass that we measure. Indeed, our mass formula is strongly reminiscent of the definition of energy in a Euclidean conformal field theory. What is more, in three dimensions we showed that naive application of a Cardy-like formula exactly reproduced the entropies of the Kerr-dS spacetimes, as also observed in $[13,14]$. However, despite this naive agreement, there are two important issues that need to be addressed-there are indications of nonunitarity (e.g., the $L_{0}$ eigenvalues are complex in general), and there is a puzzle regarding neglect of the Casimir energy contribution to the asymptotic Cardy formula.

Our results might be regarded as preliminary evidence that a Euclidean CFT dual to de Sitter space could exist. At first sight, this does not seem to jibe with the philosophy, advocated particularly by Banks, that the finite de Sitter entropy requires a finite dimensional Hilbert space $[44,45]$. However, in the picture advocated above, the finite entropy emerges because de Sitter space has a particular mass and only states of quantum gravity with this energy contribute to the entropy. This observation, and Bousso's de Sitter entropy bound [20], lead to a conjecture: Any asymptotically de Sitter spacetime with mass greater than that of de Sitter space develops a cosmological singularity. In effect, even if the Hilbert space is formally infinite dimensional, the space of initial data giving rise to de Sitter-like evolution may be finite dimensional. ${ }^{11}$

The emerging picture is that Euclidean conformal field theories do contain information about asymptotic de Sitter spaces in various sectors. A fascinating possibility is that these Euclidean theories are related to the Euclidean conformal field theories that appear in the AdS/CFT duality. Evidence in favor of this was presented in [8] where it was argued that some Euclidean de Sitter spaces have dual descriptions as sectors of the CFTs dual to AdS spaces. Further evidence, from the Chern-Simons description of 3D gravity, appeared in the work of Witten [47]. In this work, de Sitter and anti-de Sitter gravity were related by a change of the Hilbert space structure in the same underlying Chern-Simons theory. In our case, we would define a Hilbert space structure formally by cutting open the path integral of the Euclidean field theory dual to Euclidean anti-de Sitter space. The natural conjecture is that choosing a non-canonical Hilbert space structure would yield a dual to Lorentzian de Sitter space. It is very tempting to adopt this idea as a working hypothesis in trying to unearth the more precise relation between de Sitter spaces and Euclidean conformal field theories.

\section{ACKNOWLEDGMENTS}

We have benefitted from conversations with many colleagues including Tom Banks, Raphael Bousso, Robbert Dijkgraaf, Willy Fischler, Petr Hořava, Esko Keski-Vakkuri,

\footnotetext{
${ }^{11}$ We thank Tom Banks and Raphael Bousso for discussions on this issue.
} 
Dietmar Klemm, Clifford Johnson, Don Marolf, Emil Martinec, Rob Myers, Miao Li, Emil Mottola, Mu-In Park, Simon Ross, Kostas Skenderis, Andy Strominger, Erik Verlinde and Herman Verlinde. V.B. and D.M. are supported respectively by the DOE grant DE-FG02-95ER40893 and DE-FG0384ER40168. J.d.B. was also supported in part by the NSF grant PHY-9907949 at ITP, Santa Barbara. V.B. thanks the Helsinki Institute of Physics, the American University in Cairo and the University of Amsterdam for hospitality during various stages of this work. J.d.B. thanks ITP, Santa Barbara where some of this work was carried out. D.M. thanks Harvard University, the Institute for Advance Study, Princeton, the Caltech-USC Center for Theoretical Physics, the Institute for Theoretical Physics, Beijing, China and the Aspen Center for Physics for hospitality during the various stages of this work. Our results have been presented at a variety of venues including string theory workshops in Amsterdam, Beijing and Aspen.
[1] R. M. Wald, General Relativity (University of Chicago Press, Chicago, 1984); C. W. Misner, J. A. Wheeler, and K. S. Thorne, Gravitation (W. H. Freeman and Co., New York, 1973); S. Weinberg, Gravitation and Cosmology: Principles and Applications of the General Theory of Relativity (Wiley, New York, 1972).

[2] S. W. Hawking and G. F. R. Ellis, The Large Scale Structure of Space-Time (Cambridge University Press, Cambridge, England, 1973).

[3] J. D. Brown and J. W. York, Phys. Rev. D 47, 1407 (1993).

[4] D. Kastor and J. Traschen, Class. Quantum Grav. 13, 2753 (1996)

[5] D. Kastor and J. Traschen (unpublished); T. Shiromizu, D. Ida, and T. Torii, J. High Energy Phys. 11, 010 (2001).

[6] C. M. Hull, J. High Energy Phys. 07, 021 (1998); 11, 017 (1998).

[7] C. M. Hull and R. R. Khuri, Nucl. Phys. B536, 219 (1998); B575, 231 (2000).

[8] V. Balasubramanian, P. Horava, and D. Minic, J. High Energy Phys. 05, 043 (2001).

[9] E. Witten, "Quantum Gravity in de Sitter space," hep-th/0106109.

[10] A. Strominger, J. High Energy Phys. 10, 034 (2001).

[11] J. Maldacena, Adv. Theor. Math. Phys. 2, 231 (1998).

[12] E. Witten, Adv. Theor. Math. Phys. 2, 253 (1998); S. S. Gubser, Igor R. Klebanov, and Alexander M. Polyakov, Phys. Lett. B 428, 105 (1998).

[13] M. I. Park, Phys. Lett. B 440, 275 (1998).

[14] R. Bousso, A. Maloney, and A. Strominger, Phys. Rev. D 65, 104039 (2002); talk by A. Strominger at the ITP, Santa Barbara; A. Strominger and R. Bousso (private communication).

[15] D. Klemm, Nucl. Phys. B625, 295 (2002); S. Cacciatori and D. Klemm, Class. Quantum Grav. 19, 579 (2002).

[16] S. Carlip, Phys. Rev. D 51, 632 (1995); F. Lin and Y. Wu, Phys. Lett. B 453, 222 (1999).

[17] M. Banados, T. Brotz, and M. E. Ortiz, Phys. Rev. D 59, 046002 (1999).

[18] J. Maldacena and A. Strominger, J. High Energy Phys. 02, 014 (1998).

[19] S. Hawking, J. Maldacena, and A. Strominger, J. High Energy Phys. 05, 001 (2001).

[20] R. Bousso, J. High Energy Phys. 11, 038 (2000).

[21] H. Nariai, Sci. Rep. Tohoku Univ., Ser. 1 34, 160 (1950); H. Nariai, ibid. 35, 62 (1951).

[22] A. Strominger, J. High Energy Phys. 11, 072 (2001).

[23] D. Freedman, S. S. Gubser, K. Pilch, and N. Warner, Adv.
Theor. Math. Phys. 3, 363 (1999); L. Girardello, M. Petrini, M. Porrati, and A. Zaffaroni, J. High Energy Phys. 12, 022 (1998).

[24] E. Alvarez and C. Gomez, Nucl. Phys. B541, 441 (1999); V. Balasubramanian and P. Kraus, Phys. Rev. Lett. 83, 3605 (1999).

[25] J. de Boer, E. Verlinde, and H. Verlinde, J. High Energy Phys. 08, 003 (2000).

[26] V. Balasubramanian, E. Gimon, and D. Minic, J. High Energy Phys. 05, 014 (2000); V. Balasubramanian, E. Gimon, D. Minic, and J. Rahmfeld, Phys. Rev. D 63, 104009 (2001); V. Sahakian, ibid. 62, 126011 (2000).

[27] Miao Li, "Matrix Model for de Sitter," hep-th/0106184; S. Nojiri and S. D. Odintsov, Phys. Lett. B 519, 145 (2001); L. Dolan, C. R. Nappi, and E. Witten, J. High Energy Phys. 10, 016 (2001); Renata Kallosh, "N=2 Supersymmetry and de Sitter space," hep-th/0109168; C. M. Hull, J. High Energy Phys. 11, 012 (2001); 11, 061 (2001); B. McInnes, Nucl. Phys. B627, 311 (2002); R. Kallosh, A. Linde, S. Prokushkin, and M. Shmakova, Phys. Rev. D 65, 105016 (2002).

[28] L. F. Abbott and S. Deser, Nucl. Phys. B195, 76 (1982).

[29] M. Henningson and K. Skenderis, J. High Energy Phys. 07, 023 (1998).

[30] V. Balasubramanian and P. Kraus, Commun. Math. Phys. 208, 413 (1999).

[31] R. Emparan, C. V. Johnson, and R. C. Myers, Phys. Rev. D 60, 104001 (1999).

[32] S. de Haro, S. N. Solodukhin, and K. Skenderis, Commun. Math. Phys. 217, 595 (2001); K. Skenderis, Int. J. Mod. Phys. A 16, 740 (2001).

[33] P. O. Mazur and E. Mottola, Phys. Rev. D 64, 104022 (2001).

[34] C. Fefferman and C. R. Graham, "Conformal Invariants," in Asterisque 1985, p. 95.

[35] P. Kraus, F. Larsen, and R. Siebelink, Nucl. Phys. B563, 259 (1999).

[36] S. Deser and R. Jackiw, Ann. Phys. (N.Y.) 153, 405 (1984).

[37] M. Bañados, C. Teitelboim, and J. Zanelli, Phys. Rev. Lett. 69, 1849 (1992).

[38] J. D. Brown and M. Henneaux, Commun. Math. Phys. 104, 207 (1986).

[39] J. D. Bekenstein, Phys. Rev. D 7, 2333 (1973); S. W. Hawking, Commun. Math. Phys. 43, 199 (1975).

[40] G. W. Gibbons and S. W. Hawking, Phys. Rev. D 15, 2738 (1977).

[41] G. W. Gibbons and S. W. Hawking, Phys. Rev. D 15, 2752 (1977).

[42] A. Strominger, J. High Energy Phys. 02, 009 (1998). 
[43] R. Figari, R. Hoegh-Krohn, and C. R. Nappi, Commun. Math. Phys. 44, 265 (1975).

[44] T. Banks, "Cosmological breaking of supersymmetry or little Lambda goes back to the future II," hep-th/0007146.

[45] T. Banks and W. Fischler, "M-theory observables for cosmological space-times," hep-th/0102077.

[46] A. Strominger and C. Vafa, Phys. Lett. B 379, 99 (1996).

[47] E. Witten, Commun. Math. Phys. 137, 29 (1991).

[48] E. Verlinde, "On the holographic principle in a radiation dominated universe," hep-th/0008140.

[49] R. Bousso, J. High Energy Phys. 07, 004 (1999); 04, 035 (2001).

[50] G. 't Hooft, "Dimensional reduction in quantum gravity," gr-qc/9310026; L. Susskind, J. Math. Phys. 36, 6377 (1995).

[51] L. Susskind and E. Witten, "The holographic bound in anti-de Sitter space," hep-th/9805114; A. Peet and J. Polchinski, Phys. Rev. D 59, 065011 (1999).
[52] T. Yoneya, Mod. Phys. Lett. A 4, 1587 (1989); M. Li and T. Yoneya, Phys. Rev. Lett. 78, 1219 (1997). M. Li and T. Yoneya, "Short-distance space-time structure and black holes in string theory: A short review of the present status," hep-th/9806240; D. Minic, Phys. Lett. B 442, 102 (1998).

[53] P. Ginsparg and M. J. Perry, Nucl. Phys. B222, 245 (1983); see also, for example, R. Bousso, Phys. Rev. D 58, 083511 (1999); 60, 063503 (1999).

[54] J. Polchinski, Nucl. Phys. B231, 269 (1984).

[55] E. Baum, Phys. Lett. 133B, 185 (1983); S. W. Hawking, ibid. 134B, 403 (1984); S. R. Coleman, Nucl. Phys. B307, 867 (1988); B310, 643 (1988); S. B. Giddings and A. Strominger, ibid. B307, 854 (1988); B306, 890 (1988); T. Banks, ibid. B309, 493 (1988); I. R. Klebanov, L. Susskind, and T. Banks, ibid. B317, 665 (1989); P. Horava and D. Minic, Phys. Rev. Lett. 85, 1610 (2000). 